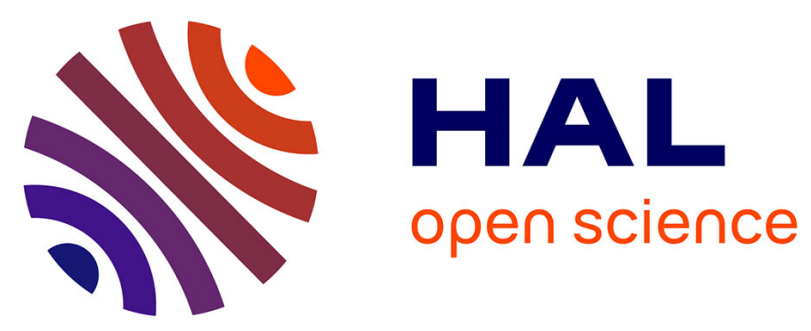

\title{
Robust Output Set-Point Tracking for a Power Flow Controller via Forwarding Design
}

Tanguy Simon, Mattia Giaccagli, Jean-François Trégouët, Daniele Astolfi, Vincent Andrieu, Xuefang Lin-Shi, Hervé Morel

\section{- To cite this version:}

Tanguy Simon, Mattia Giaccagli, Jean-François Trégouët, Daniele Astolfi, Vincent Andrieu, et al.. Robust Output Set-Point Tracking for a Power Flow Controller via Forwarding Design. CDC, Dec 2021, Austin, United States. 10.1109/CDC45484.2021.9682901 . hal-03375498

\section{HAL Id: hal-03375498 \\ https://hal.science/hal-03375498}

Submitted on 12 Oct 2021

HAL is a multi-disciplinary open access archive for the deposit and dissemination of scientific research documents, whether they are published or not. The documents may come from teaching and research institutions in France or abroad, or from public or private research centers.
L'archive ouverte pluridisciplinaire HAL, est destinée au dépôt et à la diffusion de documents scientifiques de niveau recherche, publiés ou non, émanant des établissements d'enseignement et de recherche français ou étrangers, des laboratoires publics ou privés. 


\title{
Robust Output Set-Point Tracking for a Power Flow Controller via Forwarding Design
}

\author{
Tanguy Simon ${ }^{\mathrm{a}}$, Mattia Giaccagli ${ }^{\mathrm{b}}$, Jean-François Trégoü̈t ${ }^{\mathrm{a}}$, Daniele Astolfi ${ }^{\mathrm{b}}$, Vincent Andrieu ${ }^{\mathrm{b}}$, \\ Xuefang Lin-Shi ${ }^{\mathrm{a}}$ and Hervé Morel ${ }^{\mathrm{a}}$
}

\begin{abstract}
This paper tackles the problem of robust output set-point tracking for a power flow controller (PFC) for meshed DC micro-grids. The PFC is a power electronics device used to control the power (or current) flow in the lines of the grid and act as a DC circuit breaker. The state-space model of this system is bilinear with uncertain dynamics and a polynomial output. In the proposed design, the plant is first extend with an integral action on the tracking error. The cascade model composed by the plant and the integrator is then stabilised by means of a state-feedback law, with a forwarding approach. If the plant parameters are sufficiently close to their nominal value, robust regulation is then achieved. Simulations are given to validate the results.
\end{abstract}

\section{INTRODUCTION}

Direct current (DC) micro-grids are a type of electrical network developed to enhance the penetration of renewable energy sources while lowering the energy losses and assisting the transition to a more energy-frugal lifestyle (see [1], [2] and references therein). Meshed micro-grids improve this result by requiring less copper for their wiring (since multiple paths between two points are allowed) as well as improving the modularity, reliability and efficiency of the energy system [3]. In this context, a DC power flow controller (PFC) is a multi-terminal converter located at a node of a meshed micro-grid, sometimes called a smart-node [4]. Its purpose is to regulate the power or the current in each of the lines connected to its terminals to make a better use of the meshed structure and prevent overloading.

PFCs, also denoted as current flow controllers in literature, have been widely studied for high voltage DC applications (HVDC) (see the recent survey [5]), but very little for lowvoltage applications (LVDC). Among them, recall a multiterminal PFC with a compensation node [6] improved by removing the compensation node [7], a PFC made of two separate Split-PI converters [8], and a three-terminal PFC [2]. The main difference between HVDC and LVDC PFCs resides in the topology of the converters. The latter can be designed to withstand the full line voltage and therefore they can also act as DC circuit breakers or line-voltage regulators. The main shortcomings of [6]-[8] are the absence of dynamic model and their control strategies, which fail to give any proofs of stability or robustness. No direct control of the power is achieved, and the control laws are applied to twoterminal devices, which do not constitute a node. In [6],

\footnotetext{
${ }^{a}$ Univ Lyon, INSA Lyon, Université Claude Bernard Lyon 1, Ecole Centrale de Lyon, CNRS, AMPERE, F-69621, Lyon, France

bUniversité Claude Bernard Lyon 1, CNRS, LAGEPP UMR 5007, 43 boulevard du 11 novembre 1918, F-69100, Villeurbanne, France.
}

the control law is a constant ratio determined by a lookup table, based on the knowledge of the voltage at the end of the line, an uncertain parameter in practice. In [7], a PI controller is used to regulate the current instead of the power, whose reference is again computed using the voltage at the end of the line. Moreover, the reservoir voltage is not controlled and can drift outside the physical boundaries. Finally, in [8], authors propose a current-limited voltage controller using the RST technique with hysteresis switching. The reservoir voltage is properly controlled but power flow control is not achieved. In our first approach of the problem [2], direct power flow control is achieved on a three-terminal PFC, using a state feedback on the linearised dynamics after adding integrators. This only gives local stability results in the state-space, and although its robustness has been tested, no proofs are given for local stability in the parametric space.

In this work, a PFC which is modelled as a finite dimensional state space bilinear system is studied (see Section IIA). Note that while for this class of nonlinear systems, many stabilisation techniques have been proposed (see, among them, [9]-[12] and the references therein), very few works addressed the more general problem of output regulation, e.g., [13] and more recently [14], [15]. Despite this lack of literature on output regulation, bilinear systems are a class of systems commonly employed to model physical systems (see [14], [16], [17] and many others).

In this paper, a new approach is presented for the control of the PFC. Its main improvement from existing solutions in the literature such as the multi-variable PI-controller of [2] is that the domain of attraction is semi-global in the initial conditions and local in the parametric space and that formal proofs of stability of the closed-loop system under parameters perturbations are given. The system is extended with an integral action processing the tracking error. Then, a stabiliser for the extended system is derived with a forwarding approach (see, e.g., [18], [19] or [20] for an incremental version). This control law inherits stability properties with respect to small parameter variations as shown in [19], [21], i.e., semi-global stability with regard to initial conditions holds locally in the parametric space. A similar result has been shown for DC/DC converters in [22].

The paper is organised as follows. In Section II, the model of the power flow controller is presented and the control problem is formalised. In Section III, the forwarding control design [19] is specialised for bilinear systems having a second-order polynomial output. The main results of this work are provided in Section IV. A simulation is given in 


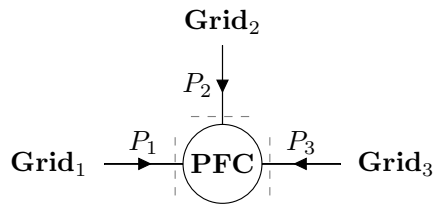

Fig. 1: The PFC at a three-terminal node in the grid, where $\mathbf{G r i d}_{k}$ represents the grid as seen by branch $k$

Section V, supporting the theoretical results. Conclusions are drawn in Section VI.

Notation. The symbol $\mathbf{I}_{m}$ stands for the identity matrix of size $m \times m$. The null matrix of size $m \times n$ is denoted by $\mathbf{0}_{m \times n}$ while $\mathbf{0}_{m}$ denotes column vectors. Dimensions are omitted when obvious from the context. The operator "diag \{\} " builds a diagonal matrix from entries of the input vector argument. Given a vector $a \in \mathbb{R}^{n}$, the notation $a_{k}$ refers to the $k$-th element of $a$, with 1 being the index of the first element. Given a square matrix $A$, $\operatorname{det}(A)$ indicates its determinant. The notation $A \succ 0(\prec 0)$ is used if $A$ is a positive (negative) definite matrix. Finally, given a set $\mathcal{S}$, $\operatorname{card}(\mathcal{S})$ denotes its cardinality and $\operatorname{int}(\mathcal{S})$ its interior.

\section{Problem Statement}

The objective of the PFC is to control the electric power in the lines it is connected to. As shown in Fig. 1, the study is here restricted to a three-terminal version and the components are assumed to be ideal to design the control law. From this illustration and knowing the PFC cannot consume or generate power, it appears clearly that the steady-state sum of powers should be zero. The chosen electrical circuit to achieve this function is made of three identical buck-boost converters connected in parallel on their high-side to a unique reservoir capacitor noted $C_{R}$. Each branch (buck-boost) is drawn on the left of Fig. 2, with the corresponding grid model on the right. This grid model is simplified in order to derive the control law.

This circuit cannot operate properly if the reservoir voltage is not controlled ${ }^{1}$. Although this adds a fourth control objective, this problem is easily solved by noting the fact that this voltage is constant if and only if the sum of powers equals zero. Consequently, if all lines except one are regulated to constant power references $P_{1}^{r}$ and $P_{2}^{r}$; and the reservoir voltage is regulated to a constant value $v_{R}^{r}$, the power in the last line will naturally converge to the overall power balance, i.e. $P_{3}^{r}$ tends to $-P_{1}^{r}-P_{2}^{r}$.

The objective of this study is to design a feedback controller delivering the duty ratios for the pulse width modulation (PWM) switching of the transistors to achieve power control in the line connected to each terminal of the PFC while maintaining the reservoir voltage to a fixed value, despite the uncertainty of the grid. Indeed, to ensure flexibility and modularity, limited knowledge is assumed on the grid model at the end of each line.

\footnotetext{
${ }^{1}$ If $v_{R}<v_{k}$, the current flows freely through the diode in $S_{H k}$, which cannot be controlled by the switching of the transistors (see Fig. 2)
}

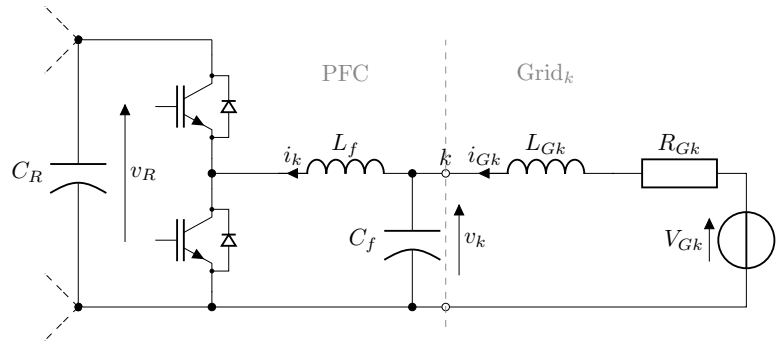

Fig. 2: Detail of the $k$-th branch of the PFC (left) and the proposed grid model as seen by this terminal (right)

\section{A. Model of The Power Flow Controller}

In Fig. 2, the currents are denoted by an $i$ and measured in Amps and the dynamic voltages by $v$ and measured in Volts. The components parameters are denoted by $L_{G k} / L_{f}, C_{R} / C_{f}$, $R_{G k}$ and $V_{G k}$ for, respectively, inductors (Henry), capacitors (Farad), resistors (Ohm) and constant voltages (Volts). A synchronous PWM switching is assumed, and the dynamics are averaged over a switching period. The input vector $\mathbf{u}=$ $\left[\begin{array}{lll}\mathbf{u}_{1} & \mathbf{u}_{2} & \mathbf{u}_{3}\end{array}\right]^{\top} \in \mathbb{R}^{3}$ is made of the three duty ratios. The dynamic variables are all gathered in the state vector $\mathbf{x} \in \mathbb{R}^{10}$ and the uncertain model parameters are gathered in the vector $\theta \in \mathbb{R}^{9}$, as shown in Tab. I. The output vector corresponds to the control objectives $\mathbf{y}=\left[\begin{array}{lll}P_{1} & P_{2} & v_{R}\end{array}\right]^{\top} \in \mathbb{R}^{3}$ (with $\left.P_{k}=i_{G k} v_{k}\right)$ and the corresponding vector of references is $\mathbf{r}=\left[\begin{array}{lll}P_{1}^{r} & P_{2}^{r} & v_{R}^{r}\end{array}\right]^{\top}$. The dynamic model of the system can be found using the electrical laws for the current and voltage in inductors and capacitors respectively while assuming ideal components (see [2] for a more detailed construction of the PFC's model). The state-space model then writes:

$$
\begin{aligned}
& \dot{\mathbf{x}}=\mathbf{A}(\theta) \mathbf{x}+\mathbf{N}(\mathbf{x}) \mathbf{u}+\mathbf{q}(\theta) \\
& \mathbf{y}=\mathbf{C} \mathbf{x}+\mathbf{H}(\mathbf{x}) \mathbf{x}
\end{aligned}
$$

with

$$
\begin{aligned}
& \mathbf{A}(\theta)=\mathbf{J}(\theta)^{-1}\left[\begin{array}{cccc}
0 & \mathbf{0}_{3}^{\top} & \mathbf{0}_{3}^{\top} & \mathbf{0}_{3}^{\boldsymbol{\top}} \\
\mathbf{0}_{3} & \mathbf{0} & \mathbf{I}_{3} & \mathbf{0} \\
\mathbf{0}_{3} & -\mathbf{I}_{3} & \mathbf{0} & \mathbf{I}_{3} \\
\mathbf{0}_{3} & \mathbf{0} & -\mathbf{I}_{3} & -\operatorname{diag}\left\{\theta_{4}, \theta_{5}, \theta_{6}\right\}
\end{array}\right] \\
& \mathbf{N}(\mathbf{x}) \mathbf{u}=\sum_{j=1}^{3}\left(\mathbf{N}_{j} \mathbf{u}_{j}\right) \mathbf{x}=\mathbf{J}(\theta)^{-1}\left[\begin{array}{ccc}
0 & \mathbf{u}^{\boldsymbol{\top}} & \mathbf{0}_{6}^{\boldsymbol{\top}} \\
-\mathbf{u} & \mathbf{0} & \mathbf{0} \\
\mathbf{0}_{6} & \mathbf{0} & \mathbf{0}
\end{array}\right] \mathbf{x} \\
& \mathbf{q}(\theta)=\mathbf{J}(\theta)^{-1}\left[\begin{array}{llll}
\mathbf{0}_{7}^{\boldsymbol{\top}} & \theta_{7} & \theta_{8} & \theta_{9}
\end{array}\right]^{\boldsymbol{\top}}, \\
& \mathbf{J}(\theta)=\operatorname{diag}\left\{C_{R}, L_{f}, L_{f}, L_{f}, C_{f}, C_{f}, C_{f}, \theta_{1}, \theta_{2}, \theta_{3}\right\} \text {, } \\
& \mathbf{C}=\left[\begin{array}{ll}
0 & \mathbf{0}_{9}^{\top} \\
0 & \mathbf{0}_{9}^{\top} \\
1 & \mathbf{0}_{9}^{\top}
\end{array}\right] \\
& \mathbf{H}(\mathbf{x})=\left[\begin{array}{ccccccc}
\mathbf{0}_{4}^{\top} & \frac{\mathbf{x}_{8}}{2} & 0 & 0 & \frac{\mathbf{x}_{5}}{2} & 0 & 0 \\
\mathbf{0}_{4}^{\top} & 0 & \frac{\mathbf{x}_{9}}{2} & 0 & 0 & \frac{\mathbf{x}_{6}}{2} & 0 \\
\mathbf{0}_{4}^{\top} & 0 & 0 & 0 & 0 & 0 & 0
\end{array}\right] \text {. }
\end{aligned}
$$

Note that the $N_{j}$ can be deduced from (2b). Name $\Theta \subset \mathbb{R}^{9}$ the non-empty set of admissible system parameters that are 
TABLE I: State vector and uncertain parameter vector.

\begin{tabular}{lllllllllll}
\hline \multirow{2}{*}{$\mathbf{x}$} & $\mathbf{x}_{1}$ & $\mathbf{x}_{2}$ & $\mathbf{x}_{3}$ & $\mathbf{x}_{4}$ & $\mathbf{x}_{5}$ & $\mathbf{x}_{6}$ & $\mathbf{x}_{7}$ & $\mathbf{x}_{8}$ & $\mathbf{x}_{9}$ & $\mathbf{x}_{10}$ \\
& $v_{R}$ & $i_{1}$ & $i_{2}$ & $i_{3}$ & $v_{1}$ & $v_{2}$ & $v_{3}$ & $i_{G 1}$ & $i_{G 2}$ & $i_{G 3}$ \\
\hline \multirow{2}{*}{$\theta$} & $\theta_{1}$ & $\theta_{2}$ & $\theta_{3}$ & $\theta_{4}$ & $\theta_{5}$ & $\theta_{6}$ & $\theta_{7}$ & $\theta_{8}$ & $\theta_{9}$ \\
& $L_{G 1}$ & $L_{G 2}$ & $L_{G 3}$ & $R_{G 1}$ & $R_{G 2}$ & $R_{G 3}$ & $V_{G 1}$ & $V_{G 2}$ & $V_{G 3}$ \\
\hline
\end{tabular}

compatible with the physics of the system, defined as

$$
\begin{array}{r}
\Theta:=\left\{\theta \in \mathbb{R}^{9}: \theta_{i}>0, i \in\{1, \ldots, 6\},\right. \\
\left.\theta_{j} \geq 0, j \in\{7,8,9\}\right\} .
\end{array}
$$

There is no loss of generality in these constraints since the first six parameters $\left(\theta_{1}, \ldots, \theta_{6}\right)$ represent physical properties (inductance and resistance), which are always strictly positive. The voltages $V_{G}$ on the line (represented by $\theta_{7}, \theta_{8}, \theta_{9}$ ) must always be non-negative. The non-empty set of admissible references is denoted $\mathcal{R} \subseteq \mathbb{R}^{3}$ and defined as

$\mathcal{R}:=\left\{\mathbf{r}=\left(P_{1}^{r}, P_{2}^{r}, v_{R}^{r}\right) \in \mathbb{R}^{3}:\left(P_{1}^{r}, P_{2}^{r}\right) \neq(0,0), v_{R}^{r}>0\right\}$

As stated before, $v_{R}^{r}$ should be sufficiently high for the device to operate properly. The case of all zero power references is not considered because it makes the control of $v_{R}$ impossible, as shown later.

\section{B. Control problem}

The issue tackled in this paper is a robust output set-point tracking problem for system (1). To this end, the system (1) is extended with an integral action of the form

$$
\dot{\mathbf{z}}=\mathbf{y}-\mathbf{r},
$$

and the aim is to stabilise the cascade formed by (1a) and (5). Indeed, as show in [19], the use of the integral action (5), is necessary and sufficient to achieve asymptotic tracking in presence of perturbations of the parameters $\theta$, when these are not perfectly known. The problem is summarised below

Problem 1. Given nominal parameters $\theta_{\text {nom }} \in \Theta$ and references $\mathbf{r}_{\text {nom }} \in \mathcal{R}$, find a state feedback control law of the form $\mathbf{u}=\alpha(\mathbf{x}, \mathbf{z})$ for the extended system (1), (5) such that the following holds.

1) (Global set-point tracking): When $(\theta, \mathbf{r})=$ $\left(\theta_{\text {nom }}, \mathbf{r}_{\text {nom }}\right)$, for any initial condition $\mathbf{x}_{0}$ in $\mathbb{R}^{10}$, the resulting trajectory of the closed-loop system is bounded forward in time and $\lim _{t \rightarrow \infty} \mathbf{y}(t)=\mathbf{r}$.

2) (Robustness): For any arbitrary compact set $\mathcal{D} \subset \mathbb{R}^{10}$, there exists $\delta>0$ such that, for any $(\theta, \mathbf{r}) \in \Theta \times \mathcal{R}$ verifying $\left|\theta-\theta_{\text {nom }}\right| \leq \delta$ and $\left|\mathbf{r}-\mathbf{r}_{\text {nom }}\right| \leq \delta$, and for any initial condition $\mathbf{x}_{0} \in \mathcal{D}$, the resulting trajectory of the closed-loop system is bounded forward in time and $\lim _{t \rightarrow \infty} \mathbf{y}(t)=\mathbf{r}$.

The key feature of the integral action is that if there exists an equilibrium for the closed loop, at this equilibrium the tracking error is equal to 0 . To design the control law, the full knowledge of the state is assumed. This is a reasonable assumption knowing that voltages and currents are easily measured. Among the different state-feedback control techniques that can be used to solve Problem 1, this work relies on forwarding-based control laws (see [19]-[21] and the references therein). In the following, such control design is specialised for bilinear systems having a second-order polynomial output. Note that the variables in the following section are in error coordinates, as in all theoretical results (Throughout the paper, a variable can be written in absolute or in error coordinates : $\mathbf{a} \neq a$ ).

\section{FORWARDING DESIGN FOR A CLASS OF BILINEAR SYSTEMS WITH POLYNOMIAL OUTPUT}

Consider a general bilinear system having a second-order polynomial output feeding an integrator of the form

$$
\dot{x}=A x+(N(x)+B) u, \quad \dot{z}=C x+H(x) x,
$$

where $(x, z) \in \mathbb{R}^{n+p}$ is the state, $u \in \mathbb{R}^{m}$ is the control input, $A, B, C$ are matrices of suitable dimensions and $H(x)=$ $\left[\begin{array}{lll}H_{1}^{\top} x & \ldots & H_{p}^{\top} x\end{array}\right]^{\top}$ and $N(x)=\left[\begin{array}{lll}N_{1} x & \ldots & N_{m} x\end{array}\right]$. Assume $m \geq p$. The following proposition gives a statefeedback control law able to stabilise the origin of (6) with a global domain of attraction.

Proposition 1. Consider system (6) and suppose that $A$ is Hurwitz and that the matrix $C A^{-1} B$ is full rank. Pick any $Q=Q^{\top} \succ 0$, and select $P=P^{\top} \succ 0, M_{i}=M_{i}^{\top}$ such that the following equations hold

$$
\begin{aligned}
P A+A^{\top} P & =-Q, \\
M_{i} A+A^{\top} M_{i} & =\frac{1}{2}\left(H_{i}+H_{i}^{\top}\right), \quad \forall i \in\{1, \ldots, p\},
\end{aligned}
$$

and let $M_{0}=C A^{-1}$. Then, for any tuning parameter $\kappa>0$, the origin of system (6) in closed loop with $u=\psi(x, z)$, with the function $\psi: \mathbb{R}^{n+p} \rightarrow \mathbb{R}^{m}$ defined as

$$
\begin{aligned}
& \psi(x, z)=-\kappa\left[2 x^{\boldsymbol{\top}} P(N(x)+B)\right. \\
& \left.\quad-2(z-M(x))^{\boldsymbol{\top}}\left(M_{0}+2 R(x)\right)(N(x)+B)\right]^{\boldsymbol{\top}},
\end{aligned}
$$

with $R(x):=\left[\begin{array}{lll}M_{1} x & \ldots & M_{p} x\end{array}\right]^{\top}, M(x):=M_{0} x+R(x) x$, is globally asymptotically stable and locally exponentially stable.

Proof. The proof is based on a Lyapunov function construction which follows the results presented in [21, Section I]. Let $W: \mathbb{R}^{n+p} \rightarrow \mathbb{R}$ be the function

$$
W(x, z)=x^{\top} P x+(z-M(x))^{\top}(z-M(x)),
$$

where $M$ is defined in the statement of the proposition. Note that $W$ is proper and positive definite. Moreover, $M$ satisfies

$$
\begin{aligned}
\frac{\partial M}{\partial x}(x) A x & =M_{0} A x+\left[\begin{array}{c}
x^{\boldsymbol{\top}}\left(M_{1} A+A^{\top} M_{1}\right) x \\
\vdots \\
x^{\boldsymbol{\top}}\left(M_{p} A+A^{\top} M_{p}\right) x
\end{array}\right], \\
& =C x+\frac{1}{2}\left[\begin{array}{c}
x^{\boldsymbol{\top}}\left(H_{1}+H_{1}^{\top}\right) x \\
\vdots \\
x^{\boldsymbol{\top}}\left(H_{p}+H_{p}^{\top}\right) x
\end{array}\right] \\
& =C x+H(x) x .
\end{aligned}
$$


Hence, the time derivative of $W$ along the solutions of System (6) satisfies,

$$
\begin{aligned}
\dot{W}(x, z)= & 2 x^{\top} P[A x+(N(x)+B) u] \\
& +2(z-M(x))^{\boldsymbol{\top}}\left[-\frac{\partial M}{\partial x}(x)(N(x)+B) u\right] \\
\leq & -x^{\top} Q x+\left[2 x^{\top} P(N(x)+B)\right. \\
& \left.-2(z-M(x))^{\boldsymbol{\top}} \frac{\partial M}{\partial x}(x)(N(x)+B)\right] u .
\end{aligned}
$$

Together with the definition of function $M$ and the control law (9), this gives

$$
\dot{W}(x, z) \leq-x^{\top} Q x-\frac{1}{\kappa} \psi^{\top}(x, z) \psi(x, z) .
$$

Note that

$$
\{(x, z): \dot{W}(x, z)=0\} \subset\{(x, z): x=0, \psi(x, z)=0\} .
$$

On the other hand, $\psi^{\top}(0, z)=2 \kappa z^{\top} C A^{-1} B$. Since by assumption, $C A^{-1} B$ is full rank and $\kappa>0$, it yields that $(x, z) \mapsto \dot{W}(z, x)$ is negative definite. Consequently, $W$ is a Lyapunov function of the closed loop system and the origin is globally and asymptotically stable. Finally, employing the same method, note that the quadratic function

$$
W_{0}(x, z)=x^{\top} P x+\left(z-M_{0} x\right)^{\top}\left(z-M_{0} x\right),
$$

is a Lyapunov function for the first order approximation

$$
\dot{x}=A x+B \psi_{0}(x, z), \quad \dot{z}=C x,
$$

with $\psi_{0}(x, z)$ being the first order approximation of $\psi$. Hence, local exponential stability of the equilibrium is obtained.

\section{MAin Results}

\section{A. Set of solutions}

Problem 1 may not be solvable for all $\theta_{\text {nom }} \in \Theta$ and references $\mathbf{r}_{\text {nom }} \in \mathcal{R}$. Indeed, the admissible parameters and references should be restricted to those for which there exists an equilibrium point $\mathbf{x}^{\star}$ on which the output $\mathbf{y}$ reaches the reference $\mathbf{r}$. In the most general case, such $\mathbf{x}^{\star}$ does not exist for any possible value of $(\theta, \mathbf{r})$ due to the nonlinear behaviour of the model. For a given $(\theta, \mathbf{r}) \in \Theta \times \mathcal{R}$, let us define beforehand the set $\mathcal{E}(\theta, \mathbf{r})$ of controlled equilibrium points, namely the set of steady state solutions for which output tracking is obtained, as

$$
\begin{aligned}
\mathcal{E}(\theta, \mathbf{r}) & :=\left\{\left(\mathbf{x}^{\star}, \mathbf{u}^{\star}\right) \in \mathbb{R}^{10} \times \mathbb{R}^{3}: \mathbf{A}(\theta) \mathbf{x}^{\star}\right. \\
& \left.+\mathbf{N}\left(\mathbf{x}^{\star}\right) \mathbf{u}^{\star}+\mathbf{q}(\theta)=0, \quad \mathbf{C x}^{\star}+\mathbf{H}\left(\mathbf{x}^{\star}\right) \mathbf{x}^{\star}=\mathbf{r}\right\} .
\end{aligned}
$$

$\mathcal{S}$ is then defined as the set of admissible parameters and references $(\theta, \mathbf{r})$ for which there exists such equilibrium points:

$$
\mathcal{S}:=\{(\theta, \mathbf{r}) \in \Theta \times \mathcal{R}: \operatorname{card}(\mathcal{E}(\theta, \mathbf{r}))>0\} .
$$

The following proposition (whose proof has been omitted for space reasons) shows that it is possible to obtain a characterisation of $\mathcal{S}$ and an expression of $\mathcal{E}$ through model inversion.

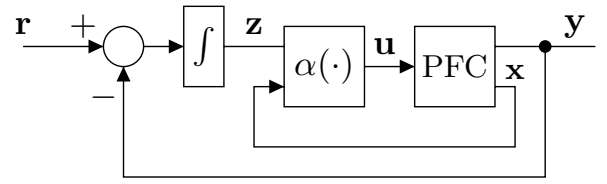

Fig. 3: Proposed control structure for the PFC

Proposition 2. Consider system (1). Then

$$
\mathcal{S}=\left\{(\theta, \mathbf{r}) \in \Theta \times \mathcal{R}: \Delta_{k}(\theta, \mathbf{r}) \geq 0, k \in\{1,2,3\}\right\},
$$

where

$$
\Delta_{k}(\theta, \mathbf{r}):=\theta_{k+6}^{2}-4 \theta_{k+3} P_{k}^{r}
$$

with $P_{3}^{r}:=-P_{1}^{r}-P_{2}^{r}$. Moreover, for a given $(\theta, \mathbf{r}) \in \mathcal{S}$, there exists from one to six pairs $\left(\mathbf{x}^{\star}, \mathbf{u}^{\star}\right) \in \mathcal{E}(\theta, \mathbf{r})$, where

$$
\mathcal{E}(\theta, \mathbf{r})=\left\{\begin{array}{l}
\mathbf{x}_{k+4}^{\star}=\frac{1}{2}\left(\theta_{k+6} \pm \sqrt{\Delta_{k}(\theta, r)}\right) \\
\mathbf{x}_{k+1}^{\star}=\mathbf{x}_{k+7}^{\star}=\frac{1}{\theta_{k+3}}\left(\theta_{k+6}-\mathbf{x}_{k+4}^{\star}\right) \\
\mathbf{x}_{1}^{\star}=v_{R}^{r} \\
\mathbf{u}_{k}^{\star}=\frac{\mathbf{x}_{k+4}^{\star}}{v_{R}^{r}}
\end{array}\right.
$$

\section{B. Controller design}

The aim of this section is to solve the output set-point tracking problem presented in Section II-A for the PFC (1) using the control structure presented in Fig. 3. The controller will be designed for some known nominal parameters and references $\left(\theta_{\text {nom }}, \mathbf{r}_{\text {nom }}\right)$. Furthermore, it is assumed that this pair belongs to $\operatorname{int}(\mathcal{S})$ rather then the whole set $\mathcal{S}$ for robustness reasons. Indeed the robustness of the proposed control law will be proven by showing that the output setpoint tracking still admits a solution for $(\theta, \mathbf{r})$ distinct from $\left(\theta_{\text {nom }}, \mathbf{r}_{\text {nom }}\right)$ but sufficiently close to it, as specified later on.

Theorem 1. Consider system (1) with $(\theta, \mathbf{r}) \in \operatorname{int}(\mathcal{S})$. Then, for any $\left(\theta_{\text {nom }}, \mathbf{r}_{\text {nom }}\right) \in \operatorname{int}(\mathcal{S})$ and any $\left(\mathbf{x}_{\text {nom }}^{\star}, \mathbf{u}_{\text {nom }}^{\star}\right) \in$ $\mathcal{E}\left(\theta_{\text {nom }}, \mathbf{r}_{\text {nom }}\right)$, the control law

$$
\begin{aligned}
& \dot{\mathbf{z}}=\mathbf{y}-\mathbf{r} \\
& \mathbf{u}=\alpha(\mathbf{x}, \mathbf{z}):=\mathbf{u}_{\text {nom }}^{\star}+\psi\left(\mathbf{x}-\mathbf{x}_{\text {nom }}^{\star}, \mathbf{z}\right)
\end{aligned}
$$

solves Problem 1, where the function $\psi$ is designed according to Proposition 1, namely of the form (9) and with the matrices $A, B, C$ and functions $N, H$ defined as

$$
\begin{aligned}
& A:=\mathbf{A}\left(\theta_{\text {nom }}\right)+\sum_{j=1}^{3} \mathbf{N}_{j} \mathbf{u}_{\text {nom }, \mathrm{j}}^{\star}, \quad B:=\mathbf{N}\left(\mathbf{x}_{\text {nom }}^{\star}\right) \\
& C:=\mathbf{C}+2 \mathbf{H}\left(\mathbf{x}_{\text {nom }}^{\star}\right), \quad N(x):=\mathbf{N}(x), \quad H(x):=\mathbf{H}(x),
\end{aligned}
$$

and $P, M$ defined as in (7), (8) and any $\kappa>0$.

Proof. The proof is divided into two parts. First, it is shown that the controller (16) achieves output set-point tracking for $(\theta, \mathbf{r})=\left(\theta_{\text {nom }}, \mathbf{r}_{\text {nom }}\right)$ and therefore satisfies the item 1 of Problem 1. Then, it is shown that the design is robust to model parameter variations, namely, for any compact set $\mathcal{D} \subset \mathbb{R}^{10}$, if $(\theta, \mathbf{r})$ is sufficiently close to $\left(\theta_{\text {nom }}, \mathbf{r}_{\text {nom }}\right)$ (as specified later on), set-point tracking is obtained for any 
initial condition $\mathbf{x}(0) \in \mathcal{D}$, thus addressing the item 2 of Problem 1.

Part 1: Global set-point tracking. Consider $(\theta, \mathbf{r})=$ $\left(\theta_{\text {nom }}, \mathbf{r}_{\text {nom }}\right) \in \operatorname{int}(\mathcal{S})$ and $\left(\mathbf{x}^{\star}, \mathbf{u}^{\star}\right) \in \mathcal{E}(\theta, \mathbf{r})$. The system (1) can then be rewritten in error coordinates $\mathbf{u} \mapsto u:=$ $\mathbf{u}-\mathbf{u}^{\star}, \mathbf{x} \mapsto x:=\mathbf{x}-\mathbf{x}^{\star}$ and $\mathbf{z} \mapsto z:=\mathbf{z}-\mathbf{z}^{\star}$, where $\mathbf{z}^{\star}=0$. In these coordinates, the system reads

$$
\begin{aligned}
\dot{x} & =\mathbf{A}(\theta)\left(x+\mathbf{x}^{\star}\right)+\mathbf{N}\left(x+\mathbf{x}^{\star}\right)\left(u+\mathbf{u}^{\star}\right)+\mathbf{q}(\theta), \\
& =\left(\mathbf{A}(\theta)+\sum_{j=1}^{3} \mathbf{N}_{j} \mathbf{u}_{j}^{\star}\right) x+\mathbf{N}\left(x+\mathbf{x}^{\star}\right) u
\end{aligned}
$$

while the $z$-dynamics read

$$
\dot{z}=\left(\mathbf{C}+2 \mathbf{H}\left(\mathbf{x}^{\star}\right)\right) x+\mathbf{H}(x) x .
$$

where the relations $\mathbf{C x}^{\star}+\mathbf{H}\left(\mathbf{x}^{\star}\right) \mathbf{x}^{\star}=\mathbf{r}$ and $\mathbf{H}(x) \mathbf{x}^{\star}=$ $\mathbf{H}\left(\mathbf{x}^{\star}\right) x$ have been used. By selecting the matrices $A, B, C$ and the functions $N, H$ as in the statement of the theorem, a system in the form (6) can be obtained. Before directly applying the result of Proposition 1, the following two technical lemmas are required, showing that $A$ is Hurwitz and that the non-resonance condition $C A^{-1} B$ full rank holds. The proofs are omitted for space reason.

Lemma 1. Pick any $(\theta, \mathbf{r}) \in \operatorname{int}(\mathcal{S})$. Then for all $\left(\mathbf{x}^{\star}, \mathbf{u}^{\star}\right) \in$ $\mathcal{E}(\theta, \mathbf{r})$, the matrix $A=\mathbf{A}(\theta)+\sum_{j=1}^{3} \mathbf{N}_{j} \mathbf{u}_{j}^{\star}$ is Hurwitz.

Lemma 2. Pick any $(\theta, \mathbf{r}) \in \operatorname{int}(\mathcal{S})$. Then for all $\left(\mathbf{x}^{\star}, \mathbf{u}^{\star}\right) \in$ $\mathcal{E}(\theta, \mathbf{r})$ the matrix $C A^{-1} B$ is full rank.

Remark 1. Lemma 1 can be understood as the natural stability of the system: in practice, for any constant duty ratio, the system stabilises to a steady-state. Concerning Lemma 2, it has been shown in [19] that such condition is necessary to achieve output set-point tracking. Since the linearized model around the equilibrium point is stabilizable, this condition implies the controllability of the extended linearised plant and it is commonly referred to as "nonresonance condition". It can be proven that the points $(\theta, \mathbf{r}) \in \partial \mathcal{S}:=\mathcal{S} \backslash \operatorname{int}(\mathcal{S})$ do not satisfy such condition.

Hence, given $(\theta, \mathbf{r}) \in \operatorname{int}(\mathcal{S})$, any $\left(\mathbf{x}^{\star}, \mathbf{u}^{\star}\right) \in \mathcal{E}(\theta, \mathbf{r})$ can be selected. Since all the assumptions are verified, the results of Proposition 1 can be applied and the control law (16) where $\psi$ is defined as in (9) for system (6), makes the equilibrium globally asymptotically stable and locally exponentially stable for the closed loop system. Moreover, on the equilibrium $\left(\mathbf{x}^{\star}, \mathbf{u}^{\star}\right), \mathbf{y}=\mathbf{r}$. Hence item 1 of Problem 1 is solved.

Part 2: Robust set-point tracking. Let $\mathbf{w}=(\mathbf{x}, \mathbf{z})$ in $\mathbb{R}^{13}$ and

$$
\dot{\mathbf{w}}=\varphi\left(\mathbf{w}, \theta_{\mathrm{nom}}, \mathbf{r}_{\mathrm{nom}}\right)
$$

be the system (1) in closed-loop with the control law (16). From item 1, system (18) admits a globally asymptotically stable and locally exponentially stable equilibrium denoted as $\mathbf{w}^{\star}$. For each compact set $\mathcal{D}$ in $\mathbb{R}^{10}$, there exists two compact sets of initial condition containing the origin and denoted $\underline{\mathcal{C}}$ and $\overline{\mathcal{C}}$ both in $\mathbb{R}^{13}$ with $\mathbf{w}^{\star} \in \underline{\mathcal{C}} \subsetneq \overline{\mathcal{C}}, \mathcal{D} \times\{0\} \subseteq \overline{\mathcal{C}}$, such that $\overline{\mathcal{C}}$ is forward invariant ${ }^{2}$ for the system (18). Therefore, by [19, Lemma 5] there exists $\rho>0$ such that, for each $C^{1}$ vector field $\varphi_{p}: \mathbb{R}^{13} \rightarrow \mathbb{R}^{13}$ satisfying

$$
\begin{aligned}
&\left|\varphi_{p}(\mathbf{w})-\varphi\left(\mathbf{w}, \theta_{\text {nom }}, \mathbf{r}_{\text {nom }}\right)\right| \leq \rho, \quad \forall \mathbf{w} \in \overline{\mathcal{C}}, \\
&\left|\frac{\partial \varphi_{p}}{\partial \mathbf{w}}(\mathbf{w})-\frac{\partial \varphi}{\partial \mathbf{w}}\left(\mathbf{w}, \theta_{\text {nom }}, \mathbf{r}_{\text {nom }}\right)\right| \leq \rho, \quad \forall \mathbf{w} \in \underline{\mathcal{C}},
\end{aligned}
$$

there exists an exponentially stable equilibrium of

$$
\dot{\mathbf{w}}=\varphi_{p}(\mathbf{w}),
$$

the basin of attraction of which contains the compact set $\overline{\mathcal{C}}$. Let us define now the function $\bar{\rho}: \Theta \times \mathcal{R} \rightarrow \mathbb{R}_{+}$as

$$
\begin{aligned}
\bar{\rho}(\theta, \mathbf{r}):=\max _{\mathbf{w} \in \overline{\mathcal{C}},\{}\left\{\left|\varphi(\mathbf{w}, \theta, \mathbf{r})-\varphi\left(\mathbf{w}, \theta_{\text {nom }}, \mathbf{r}_{\text {nom }}\right)\right|,\right. \\
\left.\left|\frac{\partial \varphi}{\partial \mathbf{w}}(\mathbf{w}, \theta, \mathbf{r})-\frac{\partial \varphi}{\partial \mathbf{w}}\left(\mathbf{w}, \theta_{\text {nom }}, \mathbf{r}_{\text {nom }}\right)\right|\right\} .
\end{aligned}
$$

Such a function is continuous and $\bar{\rho}\left(\theta_{\text {nom }}, \mathbf{r}_{\text {nom }}\right)=0$. Select now $\delta>0$, as any positive real number such that, if $\mid \theta-$ $\theta_{\text {nom }} \mid \leq \delta$ and $\left|\mathbf{r}-\mathbf{r}_{\text {nom }}\right| \leq \delta$, then $\bar{\rho}(\theta, \mathbf{r}) \leq \rho$. This parameter $\delta>0$ is a solution to the second part of Problem 1. Indeed, for each $(\theta, \mathbf{r})$ such that $\left|\theta-\theta_{\text {nom }}\right| \leq \delta$ and $\mid \mathbf{r}-$ $\mathbf{r}_{\text {nom }} \mid \leq \delta$ then the closed loop system

$$
\dot{\mathbf{w}}=\varphi(\mathbf{w}, \theta, \mathbf{r})
$$

satisfies (19) and (20) and consequently admits an exponentially stable equilibrium with a basin of attraction containing $\mathcal{D} \times\{0\}$. On this equilibrium, thanks to the integral action, output set-point tracking is achieved and this concludes the proof.

\section{Simulations}

In the following, simulation results of model (1) in closed-loop with controller (16) are shown, where the matrices $P, M_{1}, M_{2}$ and $M_{3}$ in Proposition 1 are numerically computed. Since the input $\mathbf{u}$ is made of duty ratios, in practice, $\mathbf{u}_{i} \in[0,1]$. To this end and after different simulations, a good value for the tuning parameter has been found as $\kappa=10^{-5}$. This leads to a very slow regulation for $v_{R}$, which can then be corrected by modifying the output equation with the factor $\varepsilon=5$ so that the objective becomes $\varepsilon v_{R} \rightarrow \varepsilon v_{R}^{r}$ instead of $v_{R} \rightarrow v_{R}^{r}$. The converter components $C_{R}, L_{f}$ and $C_{f}$ have values $60 \mu \mathrm{F}, 680 \mu \mathrm{H}$ and $20 \mu \mathrm{F}$, respectively. The simulation is initialised with the set-point $\left(\theta_{\text {nom }}, \mathbf{r}_{\text {nom }}\right)$ (see Tab. II), which gives the desired equilibrium states $\mathbf{x}^{\star}=$ $[500,-0.1,-1.24,2.26,402.6,400.8,398.8,-1,-1.25,2.26]$. The initial states correspond to the converter with precharged capacitors: $\mathbf{x}(0)=\left[500, \mathbf{0}_{1 \times 3}, 400 \cdot \mathbf{1}_{1 \times 3}, \mathbf{0}_{1 \times 3}\right]$. The set-point is arbitrarily changed to $\left(\theta_{a}, \mathbf{r}_{\text {nom }}\right)$ at $t=40 \mathrm{~ms}$ and then to $\left(\theta_{a}, \mathbf{r}_{a}\right)$ at $t=80 \mathrm{~ms}$. Parameters variation are highlighted in bold in Tab. II. The resulting outputs are displayed in Fig. 4, with the inputs in Fig. 5 to check that the duty ratios are well contained in $[0,1]$, including during the start-up phase. It can be seen that each

\footnotetext{
${ }^{2}$ Simply pick $\overline{\mathcal{C}}=\left\{(x, z): W(x, z) \leq c_{0}\right\}$ for sufficiently large $c_{0}$ where $W$ is a Lyapunov function for system (18).
} 
TABLE II: Set-point numerical values

\begin{tabular}{|c|c|c|c|c|c|c|c|c|c|}
\hline & Parameter & & est val & & Unit & & Parameter & Test Value & Unit \\
\hline & & $k=1$ & $k=2$ & $k=3$ & & & & & \\
\hline & $L_{G k}$ & 60 & 30 & 15 & $\mu \mathrm{H}$ & & $P_{1}^{r}$ & -400 & $\mathrm{~W}$ \\
\hline$\theta_{\text {nom }}$ & $R_{G k}$ & 2.6 & 30.3 & 1.4 & $\Omega$ & $r_{\text {nom }}$ & $P_{2}^{r}$ & -500 & W \\
\hline & $V_{G k}$ & 400 & 363 & 402 & V & & $v_{R}^{r}$ & 500 & $\mathrm{~V}$ \\
\hline & $L_{G k}$ & 60 & 30 & 30 & $\mu \mathrm{H}$ & & $P_{1}^{r}$ & -100 & W \\
\hline$\theta_{a}$ & $R_{G k}$ & 2.6 & 30.3 & 0.5 & $\Omega$ & $r_{a}$ & $P_{2}^{r}$ & -250 & W \\
\hline & $V_{G k}$ & 400 & 363 & 420 & V & & $v_{R}^{r}$ & 500 & V \\
\hline
\end{tabular}
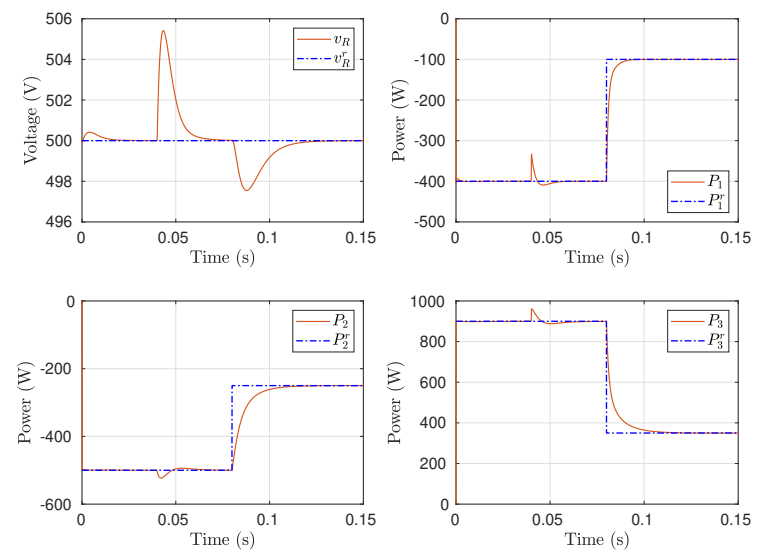

Fig. 4: Simulation of the PFC model (1) in closed loop with control (16). Reservoir voltage and power in each line with their references

reference is properly tracked. The power in the third line is also displayed to show that it is tracked properly as well, in accordance with the discussion in the introduction of Section II.

\section{CONCLUSION}

A robust output set-point tracking control law has been proposed for a 3-branch power flow controller modelled as a bilinear system with second order polynomial outputs. The control law is composed of an integral action processing the regulation error and a stabiliser for the resulting cascade system designed via forwarding techniques, ensuring a semiglobal domain of attraction and robustness to small parameters variation. Future work will concern a generalisation of the control law for any $m$-branch model, the formalisation of an input saturation, a deeper analysis of the robustness properties in the parametric space and the validation of the control scheme with experimental results.

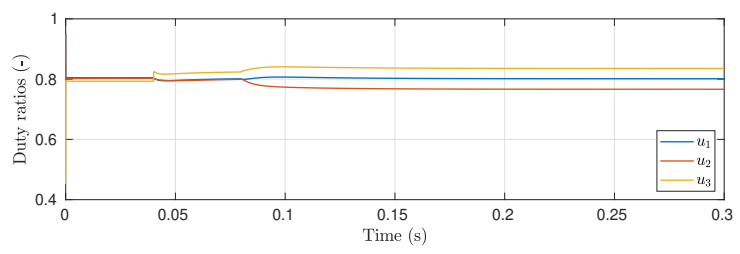

Fig. 5: Duty ratios during the simulation

\section{REFERENCES}

[1] S. Sen and V. Kumar, "Microgrid control: A comprehensive survey," Annual Reviews in control, vol. 45, pp. 118-151, 2018.

[2] T. Simon, J.-F. Trégouët, H. Morel, and X. Lin-Shi, "Modelling and control of a power flow controller for dc microgrids," in 2021 23nd European Conference on Power Electronics and Applications (EPE'21 ECCE Europe), 2021.

[3] L. Mackay, T. Hailu, L. Ramirez-Elizondo, and P. Bauer, "Decentralized current limiting in meshed dc distribution grids," in 2015 IEEE First International Conference on DC Microgrids (ICDCM). IEEE, 2015, pp. 234-238.

[4] I. Zafeiratou, I. Prodan, L. Lefèvre, and L. Piétrac, "Dynamical modelling of a dc microgrid using a port-hamiltonian formalism,' IFAC-PapersOnLine, vol. 51, no. 2, pp. 469-474, 2018.

[5] S. Balasubramaniam, C. E. Ugalde-Loo, and J. Liang, "Series current flow controllers for dc grids," IEEE Access, vol. 7, pp. 14 779-14 790, 2019.

[6] Y. Takahashi, K. Natori, and Y. Sato, "A multi-terminal power flow control method for next-generation dc power network," in 2015 IEEE Energy Conversion Congress and Exposition (ECCE). IEEE, 2015, pp. 6223-6230.

[7] K. Natori, Y. Nakao, and Y. Sato, "A novel control approach to multiterminal power flow controller for next-generation dc power network," in 2018 International Power Electronics Conference (IPEC-Niigata 2018-ECCE Asia). IEEE, 2018, pp. 588-592.

[8] M. Barara, H. Morel, and C. Guy, "Control strategy scheme for consistent power flow control in meshed de micro-grids," in COSYS$D C$, International Conference on Components and Systems do DC Grids. Grenoble, France, 2017.

[9] D. Koditschek and K. Narendra, "Stabilizability of second-order bilinear systems," IEEE Transactions on Automatic Control, vol. 28 , no. 10, pp. 987-989, 1983.

[10] P.-O. Gutman, "Stabilizing controllers for bilinear systems," IEEE Transactions on Automatic Control, vol. 26, no. 4, pp. 917-922, 1981.

[11] S. Tarbouriech, I. Queinnec, T. Calliero, and P. Peres, "Control design for bilinear systems with a guaranteed region of stability: An lmibased approach," in 2009 17th Mediterranean Conference on Control and Automation. IEEE, 2009, pp. 809-814.

[12] V. Andrieu and S. Tarbouriech, "Global asymptotic stabilization for a class of bilinear systems by hybrid output feedback," IEEE Transactions on Automatic Control, vol. 58, no. 6, pp. 1602-1608, 2012.

[13] O. M. Grasselli, A. Isidori, and F. Nicolo, "Output regulation of a class of bilinear systems under constant disturbances," Automatica, vol. 15, no. 2, pp. 189-195, 1979.

[14] R. Cisneros, M. Pirro, G. Bergna, R. Ortega, G. Ippoliti, and M. Molinas, "Global tracking passivity-based pi control of bilinear systems: Application to the interleaved boost and modular multilevel converters," Control Engineering Practice, vol. 43, pp. 109-119, 2015.

[15] G. Tang, Y. Zhao, and H. Ma, "Optimal output tracking control for bilinear systems," Transactions of the Institute of Measurement and Control, vol. 28, no. 4, pp. 387-397, 2006.

[16] B. Zitte, B. Hamroun, D. Astolfi, and F. Couenne, "Robust control of a class of bilinear systems by forwarding: Application to counter current heat exchanger," in 21st IFAC World Congress, 2020.

[17] P. M. Pardalos and V. A. Yatsenko, Optimization and Control of Bilinear Systems: Theory, Algorithms, and Applications. Springer Science \& Business Media, 2010, vol. 11.

[18] F. Mazenc and L. Praly, "Adding integrations, saturated controls, and stabilization for feedforward systems," IEEE Transactions on Automatic Control, vol. 41, no. 11, pp. 1559-1578, 1996.

[19] D. Astolfi and L. Praly, "Integral action in output feedback for multi-input multi-output nonlinear systems," IEEE Transactions on Automatic Control, vol. 62, no. 4, pp. 1559-1574, 2016.

[20] M. Giaccagli, D. Astolfi, V. Andrieu, and L. Marconi, "Sufficient conditions for output reference tracking for nonlinear systems: a contractive approach," in 2020 59th IEEE Conference on Decision and Control (CDC). IEEE, 2020, pp. 4580-4585.

[21] L. Praly, R. Ortega, and G. Kaliora, "Stabilization of nonlinear systems via forwarding mod $\left\{L_{g} V\right\}$," IEEE Transactions on Automatic Control, vol. 46, no. 9, pp. 1461-1466, 2001.

[22] G. Beneux, D. Astolfi, P. Riedinger, J. Daafouz, and L. Grimaud, "Integral action for uncertain switched affine systems with application to dc/dc converters," in European Control Conference (ECC), 2018, pp. $795-800$ 